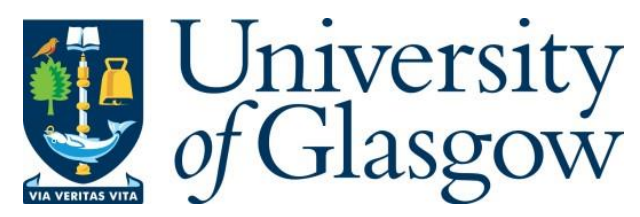

Macêdo, R., Holland, R. C., Baity, P. G., McLellan, L. J., Livesey, K. L., Stamps, R. L., Weides, M. P. and Bozhko, D. A. (2021) Electromagnetic approach to cavity spintronics. Physical Review Applied, 15(2), 024065.

There may be differences between this version and the published version. You are advised to consult the publisher's version if you wish to cite from it.

http://eprints.gla.ac.uk/234863/

Deposited on: 23 February 2021

Enlighten - Research publications by members of the University of Glasgow http://eprints.gla.ac.uk 


\title{
An Electromagnetic Approach to Cavity Spintronics
}

\author{
Rair Macêdo*, ${ }^{1}$ Rory C. Holland, ${ }^{1}$ Paul G. Baity, ${ }^{1}$ Luke J. McLellan, ${ }^{1}$ Karen L. \\ Livesey, ${ }^{2,3}$ Robert L. Stamps, ${ }^{4}$ Martin P. Weides, ${ }^{1}$ and Dmytro A. Bozhko ${ }^{3,1}$ \\ ${ }^{1}$ James Watt School of Engineering, Electronics $\mathcal{E}$ Nanoscale Engineering Division, \\ University of Glasgow, Glasgow G12 8QQ, United Kingdom* \\ ${ }^{2}$ School of Mathematical and Physical Sciences, The University of Newcastle, Callaghan NSW 2308, Australia \\ ${ }^{3}$ Center for Magnetism and Magnetic Materials, Department of Physics and Energy Science, \\ University of Colorado Colorado Springs, Colorado Springs, Colorado 80918, USA \\ ${ }^{4}$ Department of Physics and Astronomy, University of Manitoba, Winnipeg, Manitoba, MB R3T 2N2, Canada
}

(Dated: February 2, 2021)

\begin{abstract}
The fields of cavity quantum electrodynamics and magnetism have recently merged into 'cavity spintronics', investigating a quasiparticle that emerges from the strong coupling between standing electromagnetic waves confined in a microwave cavity resonator and the quanta of spin waves, magnons. This phenomenon is now expected to be employed in a variety of devices for applications ranging from quantum communication to dark matter detection. To be successful, most of these applications require a vast control of the coupling strength, resulting in intensive efforts to understanding coupling by a variety of different approaches. Here, the electromagnetic properties of both resonator and magnetic samples are investigated to provide a comprehensive understanding of the coupling between these two systems. Because the coupling is a consequence of the excitation vector fields, which directly interact with magnetisation dynamics, a highly-accurate electromagnetic perturbation theory is employed which predicts the resonant hybrid mode frequencies for any field configuration within the cavity resonator. The coupling is shown to be strongly dependent not only on the excitation vector fields and sample's magnetic properties but also on the sample's shape. These findings are illustrated by applying the theoretical framework to two distinct experiments: a magnetic sphere placed in a three-dimensional resonator, and a rectangular, magnetic prism placed on a two-dimensional resonator. The theory provides comprehensive understanding of the overall behaviour of strongly coupled systems and it can be easily modified for a variety of other systems.
\end{abstract}

\section{INTRODUCTION}

The concept of using electromagnetic waves at millimetre wavelengths trapped within resonators to probe quantum properties of matter is no stranger to us. In fact, it dates back to the 1940s when Purcell and colleagues published an abstract which was later presented at the 1946 Spring Meeting of the American Physical Society [1]. In that work, they showed that the transitions between energy levels, which correspond to different orientations of the nuclear spin in the presence of a static applied magnetic field, can couple to a resonant circuit. This coupling could then be measured through changes in the quality factor of the system. Their work was the steppingstone to the field of cavity quantum electrodynamics [2]. Interestingly enough, in that same year Griffiths also used standing waves in a microwave resonator to measure the effective high-frequency permeability of ferromagnets [3] which then led to Kittel's theory of ferromagnetic resonances [4]. More recently, these two - once distinct lines of research have come together in a newly designated area of research known as cavity spintronics which is concerned with studying 'cavity magnon-polaritons' [5-7]. These are hybrid lightmatter quasiparticles originating from the strong coupling between magnons (the quanta

\footnotetext{
* Rair.Macedo@glasgow.ac.uk
}

of spin waves) and electromagnetic waves bound inside a microwave cavity resonator [6]. One of the most fascinating aspects of these hybrid cavity-magnon systems is the potential to combine light and magnetism; and by doing so it should be possible to combine quantum information with spintronics $[8,9]$. In addition, this emergent phenomenon can also be used to engineer devices including, gradient memory devices [10], ferromagnetic haloscopes for axion detection [11-13], and radiofrequency-to-optical transducers [14].

In order to fully exploit cavity-magnon hybrid quasiparticles for applications, a deep understanding of the coupling strength is required. The coupling strength determines the degree of coherent information exchange, and thus, plays a crucial role when constructing any devices employing cavity spintronics. As an example of recent efforts into fully understanding cavity magnonpolariton coupling, we can quote Zhang and colleagues' findings[15] on the observation of exceptional points (where the two-level system's eigenfrequencies coalesce) in a cavity magnonpolariton system upon tuning the magnonphoton coupling strength. In addition, the optimisation of the coupling conditions has been shown to be a vital aspect of obtaining non-Markovian dynamics in a multi magnet-cavity hybrid system employed as a coherent, long-lifetime, broadband and multimode gradient memory with a 100-ns storage [10]. Mechanisms to control the coupling strength have so far included changing the position of the sample within the resonator [16], voltage induced control [17], as well as varying the tem- 
perature of the system [18]. More recently, a two-port cavity approach has been implemented using two-[19, 20] and three-dimensional $[21,22]$ systems as a way to achieve level attraction as well as coherent manipulation of energy exchange in the time domain [23]. These are only a few examples of the intensifying interests to fully understand and manipulate the coupling behaviour in hybrid cavity spintronic systems.

However, up to now most works have neglected how the excitation vector fields within the resonator can modify the coupling of the hybrid modes and, more importantly, how these fields directly interact with magnetisation dynamics. This includes the direction and profile of the cavity fields. A few different models have been used to describe the magnet-cavity system, one of which is the harmonic coupling model. This treats the magnet and cavity as two coupled harmonic oscillators (microscopically [5] or macroscopically [24, 25]). Another is the dynamic phase correlation model which looks at impedance changes due to charge motion generated by spin precession inside the cavity - thus relating the system to Ampéres and Faradays laws [24, 26]. While these models have captured much of the nature of hybrid cavity-spin systems, they still do not consider the full effect of complex driving fields on the spin dynamics. In addition, they also require the introduction of various experimentally-extracted parameters.

Here, we demonstrate experimentally that by modifying the position of the sample inside a resonator as well as changing the sample's shape, it is possible to drastically change the coupling strength. We explain the results with an elegant theory based on fundamental magnetic torque equation combined with Maxwell's equations. The theory is used for predicting the hybrid magnon-polariton frequencies and it shows remarkable agreement with experimental data without the use of fitting parameters or phenomenological terms such as spin density. To demonstrate that the theoretical method is generally applicable to any magnet-cavity system we use two illustrative cases: we start with a microwave cavity resonator where linearly polarised excitation is obtained, and place a magnetic Yttrium Iron Garnet $\left(\mathrm{Y}_{3} \mathrm{Fe}_{5} \mathrm{O}_{12}\right.$ or simply $\left.\mathrm{YIG}\right)[27,28]$ sphere inside. We then change the position of the sphere to exemplify how the coupling strength can be drastically modified with small changes in the microwave field profile at the sample position. Further, we investigate similar behaviour in a different cavity resonator - namely a twodimensional wave guide resonator. Using a perturbation method, we provide a theoretical framework to describe the behaviour of cavity spintronic systems based on selfconsistent electromagnetic theories. This allows for an accurate verification of our experimental findings using analytical expressions for the field profile inside the cavity and accounting for its coupling with specific magnetic permeability tensor components. This tensor is obtained from the magnetic torque equation (ie. the Landau Lifshitz equation) and can be used to treat magnets of various types and shapes. Hence, our theoretical framework is very general and can be tailored to fit a variety of different hybrid systems. Finally, we expect that by being able to fully understand the behaviour of these systems, we open up avenues for exchange and manipulation of information through cavity spintronic devices; in both classical and quantum regimes.

\section{THEORETICAL FRAMEWORK}

Before discussing our main findings, it will be necessary to revisit two well-known concepts in magnetism and microwave engineering: the response of magnetisation to an oscillating magnetic field, characterised through a dynamic susceptibility; and electromagnetic perturbation theory in a microwave resonator. These are essential for a faithful theoretical description of cavity-magnon hybridisation.

\section{A. Magnetic response through a dynamic susceptibility}

Let us start by looking at ferromagnetic resonances. This, in general, happens when a steady magnetic field, $\mathbf{H}_{\mathbf{0}}$, is applied to a spin system wherein the total magnetic moment, $\mathbf{M}$, will coherently precess about its equilibrium orientation. The behaviour can be semiclassically described by the equation of motion of magnetisation (the Landau-Lifshitz equation) [29]:

$$
\frac{\partial \mathbf{M}}{\partial t}=-\gamma \mu_{0}\left(\mathbf{M} \times \mathbf{H}_{\mathbf{e f f}}\right) .
$$

Here, the magnetisation is given in the linear approximation by $\mathbf{M}=\hat{\mathbf{z}} M_{s}+\mathbf{m} e^{j \omega t}$, with $M_{s}$ being the saturation magnetisation, $\gamma$ is the gyromagnetic ratio, and $\omega$ is the angular frequency. Note that magnetic damping is ignored for now but it will later be taken into account phenomenologically. The effective field, $\mathbf{H}_{\mathbf{e f f}}$, acting on $\mathbf{M}$ can includes contributions from the various energy terms such as Zeeman, dipole-dipole, exchange and anisotropy. Here, we consider that it contains terms due to an externally applied magnetic (d-c) field $\mathbf{H}_{\mathbf{0}}$ along the $z$ direction, an oscillating (a-c) field $\mathbf{h}$, and to account for the shape of magnetic samples we also include contributions from a demagnetising field which can be written as $\mathbf{H}_{\mathbf{D}}=-\overleftrightarrow{D} \cdot \mathbf{M}$, where $\overleftrightarrow{D}$ denotes the demagnetising tensor $\operatorname{diag}\left(D_{x}, D_{y}, D_{z}\right)[30]$. The effective field can then be written as $\mathbf{H}_{\mathbf{e f f}}=\mathbf{H}_{\mathbf{D}}+\hat{\mathbf{z}} H_{0}+\mathbf{h} e^{j \omega t}$.

In this system, resonance will occur when the oscillating magnetic field, $\mathbf{h}$, is applied with frequency $\omega$ equal to the natural frequency of precession, or the Larmor frequency, $\omega_{0}$.

Eq. (1) results in coupled, linear equations for $m_{x}$ and $m_{y}$, which in turn are used to solve for the natural fre- 
quency of precession. One requires that the amplitudes $m_{x}$ and $m_{y}$ have nontrivial solutions when there is no driving $\mathrm{rf}$ field, ie. $\mathbf{h}=0$. This yields:

$$
\omega_{0}^{2}=\gamma^{2} \mu_{0}^{2}\left[H_{0}+\left(D_{y}-D_{z}\right) M_{s}\right] \times\left[H_{0}+\left(D_{x}-D_{z}\right) M_{s}\right]
$$

To observe ferromagnetic resonance in a typical microwave experiment, one can either tune the operating frequency $\omega$ of the oscillating field $\mathbf{h}$ or the strength of the static applied field $\mathbf{H}_{\mathbf{0}}$ until the precession frequency equals the microwave frequency.

With a nonzero rf field, ie. $\mathbf{h} \neq 0$, we can write Eq. (1) in matrix form as

$$
\left[\begin{array}{l}
m_{x} \\
m_{y}
\end{array}\right]=\underbrace{\left[\begin{array}{ll}
\chi_{x x} & \chi_{x y} \\
\chi_{y x} & \chi_{y y}
\end{array}\right]}_{\overleftrightarrow{\chi}_{m}(\omega)}\left[\begin{array}{l}
h_{x} \\
h_{y}
\end{array}\right] .
$$

Here, $\overleftrightarrow{\chi}_{m}(\omega)$ is the high-frequency magnetic susceptibility which is a second rank tensor. This tensor relates the oscillating magnetisation, $\mathbf{m}$, to the oscillating magnetic field, $\mathbf{h}$ and it is often used to describe the electromagnetic response of magnetic materials.

Before looking at the behaviour of a magnet inside a microwave cavity, some intuition can be gained by exploring Eq. (3) under two different geometries, namely a ferromagnetic sphere and a rectangular prism. For this, let's take $\chi_{x x}$, which we can write as

$$
\chi_{x x}(\omega)=\frac{\chi_{a}}{1-\left(\omega / \omega_{0}\right)^{2}},
$$

where

$$
\chi_{a}=\frac{M_{s}}{H_{0}+\left(D_{x}-D_{z}\right) M_{s}} .
$$

The ferromagnetic sphere [depicted in Fig. 1(a)] is the simplest case to interpret. Due to the symmetry of the system, the demagnetising factors are the same in all directions, and thus cancel in Eq. (2). The natural frequency is simply $\omega_{0}=\gamma \mu_{0} H_{0}$ and Eq. (4) reduces to the well know form $\chi_{x x}(\omega)=\omega_{m} \omega_{0} /\left(\omega_{0}^{2}-\omega^{2}\right)$ with $\omega_{m}=\gamma \mu_{0} M_{s}$.

In the case depicted in Fig. 1(b) of a ferromagnetic rectangular prism, on the other hand, things are not so simple as the demagnetising factors are not identical [31]. In this case, both the natural resonance frequency $\omega_{0}$ and permeability tensor components have a strong dependence on the demagnetising factors as seen in Eqs. (4)-(5).

A comparison between both cases is given in Fig. 1(c) where the solid lines are for a ferromagnetic sphere and the dashed lines are for a rectangular prism. It is then evident that in both case the susceptibility component $\chi_{x x}$ has a singularity at $\omega=\omega_{0}$. However, the resonance is shifted to higher frequencies if the demagnetising fields for each direction differ from each other. Note
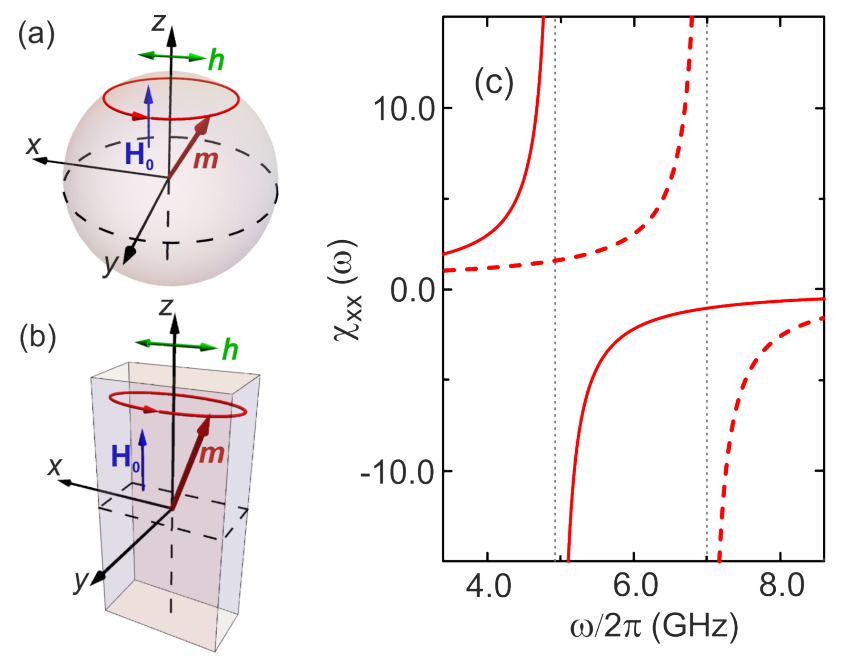

FIG. 1. Driving field, h, exciting magnetisation, m, confined to (a) a ferromagnetic sphere and (b) a ferromagnetic rectangular prism. (c) Response of $\mathbf{m}$ represented through the susceptibility tensor component $\chi_{x x}(\omega)$. The solid lines are for the sphere and the dashed lines are for the rectangular prism. The lines for $\chi_{x x}(\omega)$ were calculated using the magnetic parameters for YIG: $\mu_{0} M_{s}=0.1758 \mathrm{~T}, \gamma / 2 \pi=28 \mathrm{GHz} / \mathrm{T}$, and $\mu_{0} H_{0}=0.178 \mathrm{~T}$. Note that the resonance $\omega_{0}$ is not the same for the two systems due to different contributions of the demagnetising field. For a sphere, $\omega_{0} / 2 \pi=4.58 \mathrm{GHz}$ as all demagnetising factors are equal to $1 / 3$ due to symmetry [4]. For a rectangular prism, $\omega_{0} / 2 \pi=6.95 \mathrm{GHz}$ and the demagnetising fields were calculated [32] using the dimensions $10 \times 5 \times 50 \mu \mathrm{m}^{3}$ which yield $D_{x}=0.3266, D_{y}=0.6115$, and $D_{z}=0.0618$.

that the demagnetising factors are approximate for rectangular prisms since the demagnetising fields are in fact nonuniform [31].

It is important to point out that due to symmetry in a sphere $\chi_{x x}(\omega)=\chi_{y y}(\omega)$. This is not the case for a rectangular prism with different demagnetising factors along the $x$ and $y$ direction. This can be intuitively understood by looking at the cartoons in Fig. 1(a)-(b). For a sphere, the magnetisation is the same in $x$ and $y$, which is in stark contrast to the case of a rectangular prism as $m_{x}$ and $m_{y}$ differ from one another. Thus, one should expect that $\chi_{x x}(\omega) \neq \chi_{y y}(\omega)$. It is also worthy of note that the nonzero off-diagonal elements are well-known to give rise to various nonreciprocal effects [33-35], which are the basis for a number of important device applications [36, 37]. For completeness, all components of $\overleftrightarrow{\chi}_{m}(\omega)$ are given in Appendix A; we will not discuss these further, however, as only $\chi_{x x}(\omega)$ is used in the remainder of this work. Therefore, we will now move on to the electromagnetic perturbation theory method to describe a cavity-magnon system. 


\section{B. Perturbation Theory for Cavity Magnon-Polaritons}

In practical applications, the resonance frequency of a microwave cavity resonator, $\omega_{c}$, can be easily modified with the smallest modification in shape, size, or with a small piece of material placed inside the cavity. While the effects of these perturbations can often be difficult to quantify, they can be calculated accurately by employing perturbation theory. This holds if one assumes that the fields of a cavity with a small shape or material perturbation inside does not greatly deviate from those of the empty cavity. In recent cavity magnon-polariton experiments, a microwave cavity resonator is modified by introducing a small piece of magnetic material within the cavity. Up to now, most of the works in cavity spintronics have used approximations or oscillator models to describe the coupling and overall behaviour of the system [24, 38]. If the magnetic sample is small enough compared to the cavity volume, however, the effects of the sample and the coupling between magnon-cavity can be accurately probed using perturbation theory. A short derivation of the most general equations is presented below, and then the results for the specific geometry experimentally studied here are derived.

We start by looking at an unperturbed cavity state; that of an empty cavity, resonating in only one of its normal modes at frequency $\omega_{c}$. Let the oscillating electric and magnetic fields within the cavity be $\mathbf{E}_{\mathbf{c}}$ and $\mathbf{h}_{\mathbf{c}}$, respectively, proportional to $e^{j \omega_{c} t}$. Under these conditions, one can write Maxwell's equations as:

$$
\begin{array}{r}
\nabla \times \mathbf{h}_{\mathbf{c}}=j \omega_{c} \varepsilon_{0} \mathbf{E}_{\mathbf{c}} \\
\nabla \times \mathbf{E}_{\mathbf{c}}=-j \omega_{c} \mu_{0} \mathbf{h}_{\mathbf{c}} .
\end{array}
$$

On introducing a small ferrite sample into the cavity, the cavity will then resonate at a new frequency $\omega$ [39]. Thus, Eqs. (6a) and (6b) have to be rewritten as follows:

$$
\begin{array}{r}
\nabla \times \mathbf{h}=j \omega \varepsilon_{0} \mathbf{E}+\mathbf{J}_{\mathbf{e}} \\
\nabla \times \mathbf{E}=-j \omega \mu_{0} \mathbf{h}+\mathbf{J}_{\mathbf{m}},
\end{array}
$$

where $\mathbf{J}_{\mathbf{e}}$ and $\mathbf{J}_{\mathbf{m}}$ are the sample's dielectric and magnetic contributions which only exist in the region occupied by the perturbing material and are zero elsewhere in the cavity [37]. We can write these quantities as $\mathbf{J}_{\mathbf{e}}=j \omega \varepsilon_{0} \overleftrightarrow{\chi}_{e}(\omega) \cdot \mathbf{E}$ and $\mathbf{J}_{\mathbf{m}}=-j \omega \mu_{0} \overleftrightarrow{\chi}_{m}(\omega) \cdot \mathbf{h}$. Here $\overleftrightarrow{\chi}_{e}(\omega)$ and $\overleftrightarrow{\chi}_{m}(\omega)$ are the electric and magnetic susceptibility contributions of the ferrite respectively (both written in tensor form for a more general description). Following common vector algebraic operations [40], we can obtain the following relation:

$$
\omega-\omega_{c}=j \frac{\int_{\delta v}\left(\mathbf{J}_{\mathbf{e}} \cdot \mathbf{E}_{\mathbf{c}}^{*}-\mathbf{J}_{\mathbf{m}} \cdot \mathbf{h}_{\mathbf{c}}^{*}\right) \mathrm{d} v}{\int_{v}\left(\varepsilon_{0} \mathbf{E}_{\mathbf{c}}^{*} \cdot \mathbf{E}+\mu_{0} \mathbf{h}_{\mathbf{c}}^{*} \cdot \mathbf{h}\right) \mathrm{d} v} .
$$

where $\delta v$ is the sample volume, and $v$ is the volume of the empty cavity.

This expression is exact, given the perturbative assumptions made in Eqs. (7), and could be evaluated if the configuration of $\mathbf{E}$ and $\mathbf{h}$ for the perturbed cavity were known. In general, this can be hard to estimate. For cavity measurements in which the samples are small enough, however, one can assume that $\mathbf{E}=\mathbf{E}_{\mathbf{c}}$ and $\mathbf{h}=\mathbf{h}_{\mathbf{c}}$ everywhere inside the cavity. For simplicity, and for the remainder of this work, we can also consider that there are no dielectric contributions from the sample and it responds only to the $\mathbf{h}_{\mathbf{c}}$ field of the cavity, so that we can make $\mathbf{E}_{\mathbf{c}}=0$. This way, Eq. (8) can be rewritten as

$$
\omega-\omega_{c}=-\omega_{c} \frac{\int_{\delta v} \mu_{0}\left[\overleftrightarrow{\chi}_{m}(\omega) \cdot \mathbf{h}_{\mathbf{c}}\right] \cdot \mathbf{h}_{\mathbf{c}}^{*} \mathrm{~d} v}{2 \int_{v} \mu_{0} \mathbf{h}_{\mathbf{c}}^{*} \cdot \mathbf{h}_{\mathbf{c}} \mathrm{d} v} .
$$

\section{DEPENDENCY ON THE DISTRIBUTION OF A LINEARLY POLARISED FIELD}

We will now apply the theory detailed so far to understand the coupling between microwaves confined in cavity resonators and magnons. We will start by looking at the simple case of magnons in a YIG sphere, as depicted in Fig. 2(a), excited by microwave fields in a threedimensional, rectangular cavity, as depicted in Fig. 2(b). For this, we experimentally probed the behaviour of the hybrid system as the position of a magnetic sphere inside the cavity is changed. This allows us to gain some insight into how the coupling strength can be modified as we change the profile and configuration of the oscillating field, $\mathbf{h}_{\mathbf{c}}$, around the sample position.

Our rectangular, three-dimensional microwave resonator [see Fig. 2(b)] was machined from high conductivity aluminium to obtain a high $\mathrm{Q}$ factor and it has inner dimensions of $54 \times 36 \times 7 \mathrm{~mm}^{3}$. We have used capacitive coupling to generate a $\mathrm{TE}_{110}$ mode resonating at frequency $\omega_{c} / 2 \pi=4.98 \mathrm{GHz}$ with a linewidth of a few $\mathrm{MHz}$. The intensity profile of the oscillating magnetic field, $\mathbf{h}_{\mathbf{c}}$, calculated using COMSOL 5.5, is also shown in Fig. 2(b) with anti-node (marked as $A$ ) at $x=27 \mathrm{~mm}, y=2.5 \mathrm{~mm}$ and $z=3.75 \mathrm{~mm}$. Spectroscopic measurements were then carried out with a vector network analyzer (VNA) by probing the reflection of the microwave cavity through a coaxial cable antenna (further details on the experimental setup are given in Appendix D). The small magnetic sample (YIG sphere of diameter $0.5 \mathrm{~mm}$ ) was placed in the anti-node of $\mathbf{h}_{\mathbf{c}}$ and biased with a static field, $\mathbf{H}_{\mathbf{0}}$, externally applied along $z$, and hence perpendicular to the oscillating field $\mathbf{h}_{\mathbf{c}}$. This ensures that the ferromagnetic resonance excitation condition is met. As discussed in Sec. II A, the frequency of the ferromagnetic mode for a sphere, such as that depicted in Fig. 2(a), linearly depends on $\mathbf{H}_{\mathbf{0}}$ following the relation $\omega_{0}=\gamma H_{0}$. The bias 

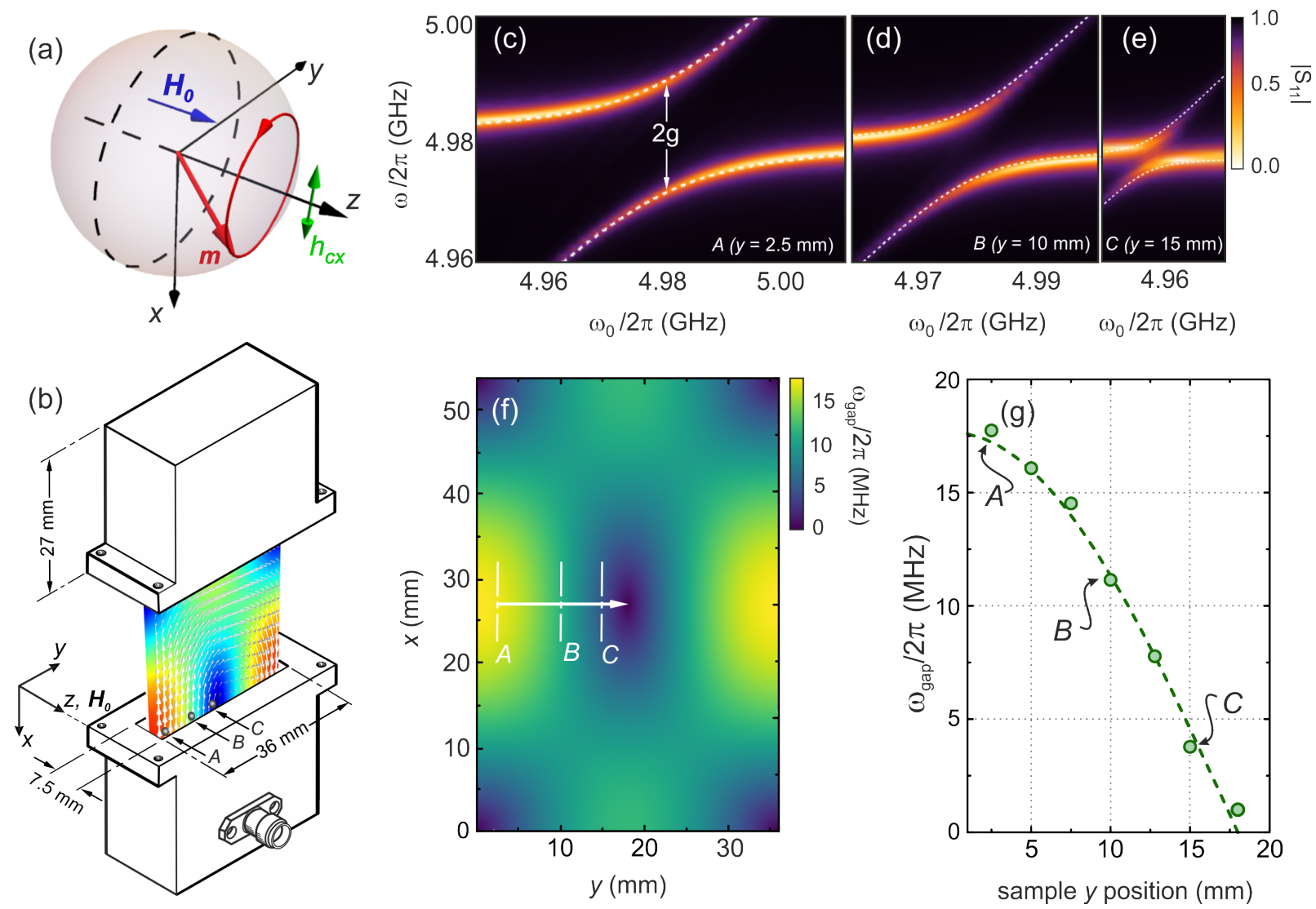

FIG. 2. (a) Behaviour of magnetisation excited by a linearly polarised excitation such as shown in (b) when the sample is placed inside of a rectangular microwave resonator. We show a cross-sectional field configuration at $z=3.75 \mathrm{~mm}$ generated by capacitive coupling (simulated with COMSOL 5.5). Experimental $\left|S_{11}\right|$ spectra (colour maps) and perturbation theory (dashed lines) for the hybridised cavity magnon-polariton modes, close to $\omega_{c}=\omega_{0}$, for a YIG sphere (0.5 mm diameter) placed at positions (c) $A(y=2.5)$, (d) $B(y=10)$, and (e) $C(y=15 \mathrm{~mm})$. In part (f) we give a full map of $\omega_{\text {gap }}$ for any given $x-y$ position. (g) Experimental points and theoretical lines of $\omega_{\text {gap }}$ as the sample is moved within the microwave cavity (along $y$ and at $x=27 \mathrm{~mm}$ ) through positions $A, B$ and $C$ [see panel (b)].

magnetic field is then swept so that $\omega_{0}$ crosses the cavity resonance frequency, $\omega_{c}$ (corresponding to fields around $0.178 \mathrm{~T})$.

The measured $\left|S_{11}\right|$ spectrum is shown in Fig. 2(c), where a strong anti-crossing between the two modes of the system is observed. This has often been referred to as level repulsion of the magnon-cavity polariton and is a classic feature of the hybridisation; analogous to a two-level system. In this case, the macroscopic coupling strength, $g$, is often associated with the width of the splitting at $\omega_{c}=\omega_{0}$ which is where the hybridized modes have their minimum frequency separation. In the strong coupling regime, these are related by $2 g=\left|\omega_{a}-\omega_{b}\right|=\omega_{\text {gap }}$ [24]. Here, $\omega_{a}$ and $\omega_{b}$ are the eigenfrequencies for the two modes (branches) seen in Fig. 2(c). Again, in analogy to a two-level system, this would correspond to the minimum value of the generalized Rabi gap [41].

In Figs. 2(d) and (e) we show the effect of moving the sample away from the anti-node of $\mathbf{h}_{\mathbf{c}}$ to positions $B$ $(y=10 \mathrm{~mm})$ and $C(y=15 \mathrm{~mm})$, respectively. The most drastic effect observed here is that $\omega_{\text {gap }}$ becomes very small as $\mathbf{h}_{\mathbf{c}}$ is close to vanishing - for reference, the positions $\mathrm{A}, \mathrm{B}$, and $\mathrm{C}$ are drawn in Fig. 2(b).

To quantify - and indeed predic - the behaviour of $\omega_{\text {gap }}$, we now employ the perturbation theory described in detail earlier. For this, we consider that in the YIG sphere used in our experiment, the effect of an applied field $\mathbf{H}_{\mathbf{0}}$ directed along $z$ is to induce precession that can only couple with the components of $\mathbf{h}_{\mathbf{c}}$ along the $x$ and/or $y$ directions, $h_{c x}$ and $h_{c y}$ respectively. If we concentrate on the behaviour of the sample moved from the anti-node to the node of $\mathbf{h}_{\mathbf{c}}$ (from $y=2.5 \mathrm{~mm}$ to point $y=18 \mathrm{~mm}$, but always at $x=27 \mathrm{~mm}$ ), we can neglect $h_{c y}$ as it is much smaller than $h_{c x}$ at all points. This means the sample is always excited by a linearly polarised field. We can then use Eq. (3) to rewrite Eq. (9) as: 


$$
\begin{aligned}
\frac{\omega-\omega_{c}}{\omega_{c}} & =-\chi_{x x}(\omega) \frac{\int_{\delta v} \mu_{0}\left|h_{c x}\right|^{2} \mathrm{~d} v}{2 \int_{v} \mu_{0} \mathbf{h}_{\mathbf{c}}^{*} \cdot \mathbf{h}_{\mathbf{c}} \mathrm{d} v} \\
& =-\frac{\omega_{0} \omega_{m}}{\omega_{0}^{2}-\omega^{2}} \frac{W_{p}}{W_{c}} .
\end{aligned}
$$

For simplicity, we write the quantities relating to the oscillating fields in Eq. (10) as $W_{c}$ and $W_{p}$, respectively. $W_{p}$ corresponds to the integral of the oscillating magnetic field across the sample volume and since we can neglect $h_{c y}$ for our positions of interest, in Eq. (10) we were able to reduce this to $W_{p}=\int_{\delta_{v}} \mu_{0}\left|h_{c x}^{2}\right| d v$ - with $h_{c x}$ being equivalent to $h_{x}$ in Eq. (3). For a more complicated field geometry involving both $\mathrm{x}$ and $\mathrm{y}$ components of $\mathbf{h}_{\mathbf{c}}$, see Apendix C. As for $W_{c}$, this represents the total energy stored in the empty cavity $\left(W_{c}=W_{m}+W_{e}\right.$, where $W_{m}$ is the total magnetic energy and $W_{e}$ is the total electric energy). In the empty cavity both $W_{m}$ and $W_{e}$ are the same, thus we can simply quote one of them, as done in the denominator of Eq. (10) where we write $W_{c}=2 W_{m}$.

Because we are interested in the behaviour at frequencies close to both the cavity and magnetic resonance frequencies, we can use the relation $\omega_{0}^{2}-\omega^{2} \approx\left(\omega_{0}-\omega\right) 2 \omega_{0}$ into Eq. (10) to find

$$
\left(\omega-\omega_{c}\right)\left(\omega-\omega_{0}\right)=\frac{1}{2} \omega_{c} \omega_{m} \frac{W_{p}}{W_{c}} .
$$

We can then solve this for $\omega$, which yields:

$$
\omega_{a, b}=\frac{1}{2}\left[\omega_{c}+\omega_{0} \pm \sqrt{\left(\omega_{c}-\omega_{0}\right)^{2}+2 \omega_{c} \omega_{m} \frac{W_{p}}{W_{c}}}\right] .
$$

These are the eigenfrequencies of the cavity-magnon hybrid system. With these equations, and using the magnetic parameters for YIG, i.e. same as those used in Fig. 1(c), we obtain the dashed lines in Fig. 2(c)-(e) which are in excellent agreement with the experimental contour data. The intensity of the field at the sample position can be calculated analytic - with full equations quoted in Appendix B. For simple rectangular cavities, such as the one shown in in Fig 2(a), $W_{c}$ can also be calculated analytically using $W_{c}=1 / 2\left(\varepsilon_{0} v\right)$. These relations can be further used to calculate the size of the splitting, which at $\omega_{c}=\omega_{0}$ is given by:

$$
\omega_{\text {gap }}=\left.\left(\omega_{a}-\omega_{b}\right)\right|_{\omega_{c}=\omega_{0}}=\sqrt{2 \omega_{c} \omega_{m} \frac{W_{p}}{W_{c}}} .
$$

The heat map shown in Fig. 2(f) summarizes the behaviour of $\omega_{g a p}$ as function of both $x$ and $y$ positions within the resonator calculated from Eq. (13). This clearly shows that the behaviour of $\omega_{\text {gap }}$ strongly reflects the intensity of $\mathbf{h}_{\mathbf{c}}$ given in Fig. 2(b). Fig. 2(g) shows how the predicted values of $\omega_{\text {gap }}$ from the analytical expressions from perturbation theory combined with analytical values for the oscillating field inside the cavity (green dashed lines) match experimental points (green dots) as the sample is moved within the resonator shown in part (b) along the $x$-axis. Note that we have also compared our analytical results with numerical simulations by evaluating $W_{c}$ and $W_{p}$ using COMSOL. We have obtained, for position $A$ in Fig. 2(b), $W_{m}=\int_{v} \mu_{0} \mathbf{h}_{\mathbf{c}}^{*} \cdot \mathbf{h}_{\mathbf{c}} \mathrm{d} v=5.55 \times 10^{-10} \mathrm{~J}$ and $\int_{\delta v} \mu_{0}\left|h_{c x}\right|^{2} \mathrm{~d} v=6.875 \times 10^{-15}$ J. These values return $W_{p} / W_{c}=6.26385 \times 10^{-6}$ which yield $\omega_{\text {gap }}=17.6 \mathrm{MHz}$. This is consistent with both analytical and experimental values shown in Fig. 2(g). Details on the analytic calculation of the field distribution are given in Appendix B.

As discussed in Sec. IIB, the perturbation theory method can be employed when the sample does not cause great deviation on the cavity fields, and hence its resonance frequency. Here, the sphere size only accounts for less than $0.005 \%$ of the cavity volume, in which case, the sample does not induce appreciable deviation from the resonance frequency of the empty cavity $\omega_{c}$. In fact, the maximum possible sphere size in our experiment $(\approx 7 \mathrm{~mm}$ diameter) would only account for a $2 \%$ deviation on the resonance frequency (we have included further details of the sample size effect in the Supplemental information [42]).

A few main remarks should be made here:

- The coupling constant has been previously estimated by various models fitting experimental data. However, as seen here, perturbation theory is an effective way to exactly calculate $g$ without any need for experimental fitting parameters such as spin density.

- In order to solve Eq. (10), it is not necessary to make any approximations as the ones made here to obtain Eq. (12). However, the approximations work well close to the splitting.

- Finally, at our initial sample position [shown as $A$ in Fig. 2(b)] the $\mathbf{h}_{\mathbf{c}}$-field within the cavity is close to its maximum value, and lies in the $\hat{\mathbf{x}}$ direction. While the oscillating field, $\mathbf{h}_{\mathbf{c}}$, gains other components as we move the sample away from the antinode it is still always linearly polarised; and thus, nothing changes for the perturbation theory. However, the coupling dramatically changes from maximal (at the anti-node of $\mathbf{h}_{\mathbf{c}}$ ) to vanishing (at the anti-node of $\mathbf{E}_{\mathbf{c}}$ ).

Furthermore, the perturbation theory described here, with some modifications, can be readily applied to microwave resonators and transmission lines of any kind by describing the field distribution. It can also be used for a variety of different magnetic samples by obtaining the appropriate permeability tensors (considering shapes, 
structuring, or composition). A case example is now given.

\section{MAGNETIC THIN-FILM STRIPES COUPLED TO A TRANSMISSION LINE RESONATOR}

Now that we have investigated a simple case, against which we have verified the validity of the perturbation theory for various field configurations, we move on to a more complicated resonator. Up to now we have discussed the case of a large and three-dimensional system, where the resonator is in the order of a few centimetres. This is often the dimensions of cavity spintronic devices to enhance the coupling rates. However, in order to easily integrated these with either silicon-based or superconducting quantum circuits, for example, it is necessary to reduce the system's dimensions towards on-chip scalable devices. The first studies on on-chip cavity spintronic devices were by Hou et al. [43] and Li et al. [44]. We will therefore now look at a similar system to the ones investigated in those studies, where a micrometresized stripe of magnetic materials is placed on planar (two-dimensional) superconducting resonators. One way to get this is by employing a coplanar waveguide structure with two gaps in the center conductor such as the schematics shown in Fig. 3(a). The gaps in the centre conductor form a planar capacitor acting as dielectric mirrors, which in turns generates standing waves. These determine the resonance frequency through the separation length between the capacitors, $l$, which is a multiple of the half wavelength $\lambda / 2[32]$.

The magnetic thin-film stripe is placed in the center of the resonator, and much like our previous case of a three-dimension system, an external magnetic field is used to set the ferromagnetic frequency of the sample near the cavity resonance frequency. However, as opposed to the case of a sphere, the magnetic precession drastically changes due to the shape of the sample, as shown in Fig. 3(b) and discussed in Sec. II A. The confined dimensions now induce highly elliptical precession behaviour and that can be quantified through demagnetising factors in the susceptibility, as discussed in Eq. (3). In order to obtain the condition for ferromagnetic resonance, the sample is positioned so that the oscillating magnetic field generated by the centre conductor is perpendicular to the static field. Here, the relevant component of the oscillating field $\mathbf{h}_{\mathbf{c}}$ at the sample position is, again, along the $x$ direction [in Fig. $3(\mathrm{~b})$ this is depicted as $\left.h_{c x}\right]$.

The more complex field profiles inside the twodimensional resonator, compared to the 3D cavity discussed earlier, only marginally affect the perturbation method described earlier. In fact, the main difference for this particular case is the calculation of the fields exciting the magnetic sample, contained in $W_{p}$, and the total energy stored in the resonator $W_{c}$ [both discussed near Eq. (12)]. These quantities can no longer be calculated analytically as we have done in previous sections, but they can easily be estimated using electromagnetic solvers such as HFSS or COMSOL (See supplemental information for details [45]). Once those are estimated, the eigenfrequencies can be obtained using:

$$
\omega_{a, b}=\frac{1}{2}\left[\omega_{c}+\omega_{0} \pm \sqrt{\left(\omega_{c}-\omega_{0}\right)^{2}+2 \chi_{a} \omega_{c} \omega_{0} \frac{W_{p}}{W_{c}}}\right]
$$

Note that this equation is slightly different from Eq. (12). This is because we now have to account for the demagnetising fields and use the full form of $\chi_{x x}$ as given in Eq. (4). The resulting anti-crossing, calculated using Eq. (14), is shown in Fig. 3(c) as the solid lines. For this, we have used $\mathrm{Ni}_{80} \mathrm{Fe}_{20}$ - also known as Permalloy $(\mathrm{Py})$ as the example material for the magnetic thin-film stripe with parameters $\mu_{0} M_{s}=1 \mathrm{~T}$ and $\gamma / 2 \pi=28 \mathrm{GHz} / \mathrm{T}$ [44]. The demagnetising parameters are $D_{x}=0.0052$, $D_{y}=0.9947$, and $D_{z}=0.00008$ [31] and the oscillating magnetic field $h_{0 x}$ at the sample position and energy stored in the system $W_{c}$ were calculated using COMSOL yielding $W_{p}=2.868 \times 10^{-15} \mathrm{~J}$ and $W_{c}=3.6 \times 10^{-11} \mathrm{~J}$ for a ration $W_{p} / W_{c}=7.967 \times 10^{-5}$.

Knowing the fields in the resonator and the dimensions of the magnetic sample, it is also straightforward to estimate the coupling constant $g$ through the width of the splitting using the relation:

$$
\omega_{g a p}=\left.\left(\omega_{a}-\omega_{b}\right)\right|_{\omega_{c}=\omega_{0}}=\sqrt{2 \chi_{a} \omega_{c} \omega_{0} \frac{W_{p}}{W_{c}}} .
$$

This yields $\omega_{g a p} / 2 \pi=0.357 \mathrm{GHz}$ or $g / 2 \pi=0.178 \mathrm{GHz}$.

\section{A. Effect of Damping on the Coupling Strength}

Our calculated coupling strength, $g$, using Eq. (15) is 26.6 $\mathrm{MHz}$ higher when compared to the case reported by $\mathrm{Li}$ and co-workers [44]. While we have used the same resonance frequency, material parameters, and sample dimensions as reported in their work, there is one property we have neglected so far: damping. This was not necessary when looking at YIG spheres, as in this case the linewidth of the magnetic resonance is small enough so that it does not affect the eighenfrequencies from perturbation theory. In magnetic thin films, however, such linewidths are not only a result of intrisinc damping but they are often broadened by various surface and interface non-uniformity as well as sample defects - known as inhomogeneous broadening. In general, the effect of damping and dissipation can be introduced by replacing $\omega_{0}$ with a complex frequency $\omega_{0} \rightarrow \omega_{0}^{\prime}+j \omega_{0}^{\prime \prime}$, or even by just replacing $H_{0}$ with a complex magnetic field $H_{0} \rightarrow H_{0}^{\prime}+j \Delta H_{0}$ where $\Delta H_{0}$ is the width of the resonance curve at half height. 

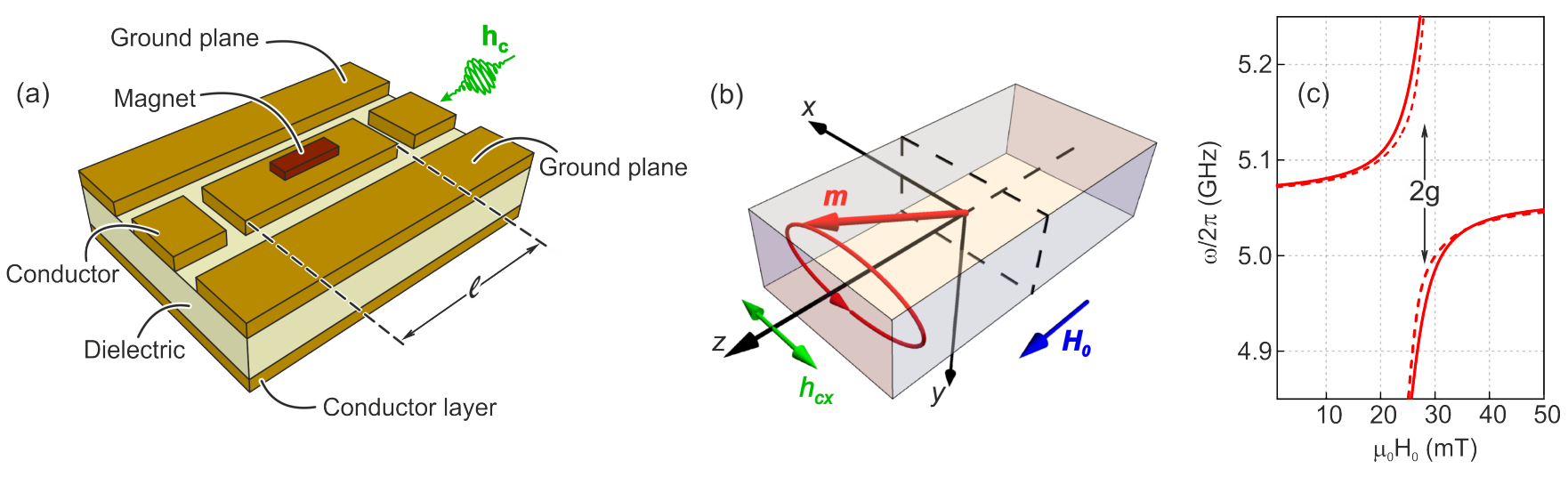

FIG. 3. (a) Diagram of the set up used here where a two-dimensional coplanar waveguide resonator generates an oscillating magnetic field which couples to oscillating magnetisation in a magnetic thin-film stripe. A static field, $\mathbf{H}_{\mathbf{0}}$ is applied along the sample's long axis (along $z$ ) and the oscillating magnetic field, $\mathbf{h}_{\mathbf{c}}$, at the sample position only has a component along the $x$ direction - denoted as $h_{c x}$. A full schematic of the magnetic sample and oscillating magnetisation with respect to the oscillating and static fields is given in (b). (c) Anti-crossing for the hybrid magnon-resonator modes calculated close to $\omega_{c}=\omega_{0}$. Here, we considered the magnetic sample to be a Py $\left(\mathrm{Ni}_{80} \mathrm{Fe}_{20}\right)$ rectangular prism $\left(14 \times 0.03 \times 900 \mu \mathrm{m}^{3}\right)$ and the resonance frequency of the resonator is $\omega_{c} / 2 \pi=5.0 \mathrm{GHz}$. The solid lines are for no damping [using Eq. (14)] and the dashed lines take damping into account.

Applying the former description into Eq. (10) we obtain the dashed lines in Fig. 3(c) using $\omega_{0}^{\prime \prime}=0.035 \mathrm{GHz}$ as measured by Li and co-workers [44]. The size of the splitting for a Py thin-film strip when dissipation is considered is clearly smaller than when no damping is taken into account. With dissipation, Eq. (14) which quantifies $\omega_{\text {gap }}$ now becomes:

$$
\begin{aligned}
\omega_{\text {gap }} & =\left.\left(\omega_{a}-\omega_{b}\right)\right|_{\omega_{c}=\omega_{0}}= \\
& \sqrt{2 \chi_{a} \omega_{c} \omega_{0}^{\prime} \frac{W_{p}}{W_{c}}+j \omega_{0}^{\prime \prime}\left(2 \chi_{a} \omega_{c} \frac{W_{p}}{W_{c}}+j \omega_{0}^{\prime \prime}\right)},
\end{aligned}
$$

and for the case shown in Fig. 3(c), we can use Eq. (16) to find $\omega_{\text {gap }} / 2 \pi=0.309 \mathrm{GHz}$ or simply $g / 2 \pi=0.154 \mathrm{GHz}$. This is in excellent agreement with the value for the coupling strength of $g / 2 \pi=0.152 \mathrm{GHz}$ reported by $\mathrm{Li}$ and co-workers [44].

\section{SCATTERING PARAMETER AND QUALITY FACTOR FROM PERTURBATION THEORY}

As we have seen so far, cavity perturbation theory in itself is an extremely efficient method to measure the coupling strength of hybrid systems. However, this technique can also be employed when computing scattering parameters. These quantities are often measured by VNAs in spectroscopic experiments, much like the data we have discussed in Fig. 2(c)-(e).

We can then employ a scattering matrix formalism in order to investigate how microwave radiation interacts with the hybrid system. In the vicinity of the resonances, the behaviour of the cavity resonator and magnet can both be represented as lumped circuits. This way we can assume that a voltage wave $a_{1}$ is incident on an arbitrary microwave device. The wave is scattered and some of its energy goes into a reflected wave $a_{2}$ and part into a transmitted wave $b_{2}$. Therefore the scattering parameter $S_{11}(\omega)$ (which we have looked at Fig. 2) is given by the ratio $a_{2} / a_{1}$ [46]. Moreover, in order to account for both resonant hybrid systems, we take into account the product of the two resonances obtained as our perturbation theory eigenfrequencies so that we can make $S_{11}(\omega)=S_{11}^{(a)}(\omega) \times S_{11}^{(b)}(\omega)$, with [47]:

$$
S_{11}^{(a, b)}(\omega)=\frac{\beta-1-j Q_{(a, b)}\left[\omega / \omega_{(a, b)}-\omega_{(a, b)} / \omega\right]}{\beta+1+j Q_{(a, b)}\left[\omega / \omega_{(a, b)}-\omega_{(a, b)} / \omega\right]} .
$$

Here, $\beta$ is the propagation constant which determines whether the system is undercoupled (taking $\beta<1$ ); overcoupled (taking $\beta>1$ ); or if the resonator is critically coupled (taking $\beta=1$ ). We also take the quality factor $Q$ to be [48]:

$$
Q_{(a, b)}=\frac{\omega_{(a, b)}^{\prime}}{\omega_{(a, b)}^{\prime \prime}},
$$

where $\omega_{(a, b)}^{\prime}$ is the real part of either eigenfrequency $a$ or $b$ calculating with the equations from perturbation theory, such as Eq. (12), and $\omega_{(a, b)}^{\prime \prime}$ denotes their equivalent imaginary part.

In Fig. 4(a) we show the calculated scattering parameter mirroring that shown in Fig. 2(c) at the node of the magnetic field inside the resonator. In Fig. 4(b) a comparison between the experimental and theoretical 

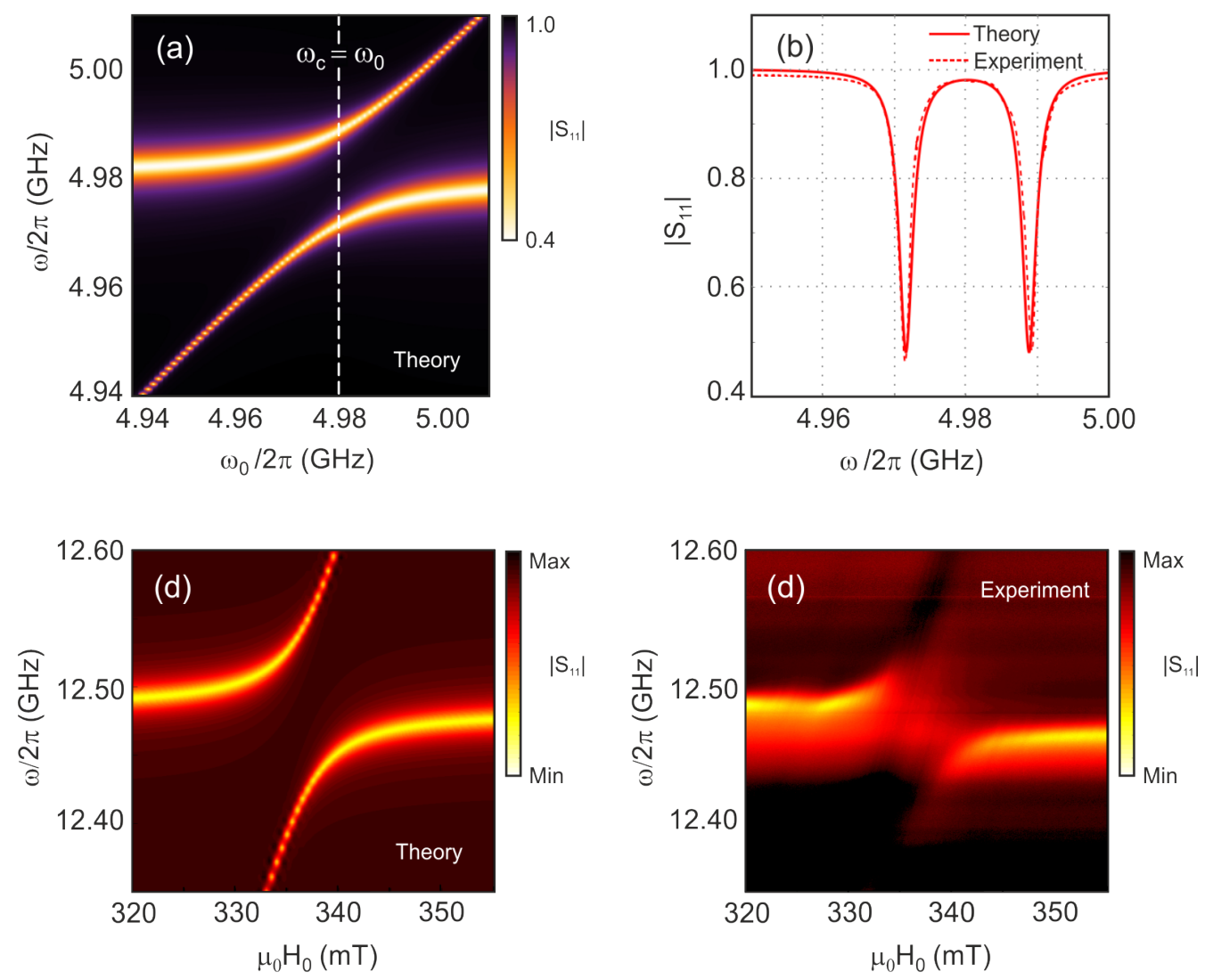

FIG. 4. (a) Scattering parameter $\left|S_{11}\right|$ calculated from the quality factor, $Q_{p}$, from perturbation theory as a function of both input frequency $\omega$ and externally applied magnetic field $\mathbf{H}_{\mathbf{0}}$. (b) Comparison between experimental and theoretical $\left|S_{11}\right|$ spectra at $\omega_{c}=\omega_{0}$. The solid line is for the theory [vertical cut in (a)] and the dashed line is for experimental data [a vertical cut in Fig. 2(b)]. Here we have considered the system to be slightly overcoupled with $\beta=1.05$; and the dissipation for the two systems were taken to be $\omega_{c}^{\prime \prime}=1.3 \times 10^{-3}$ and $\omega_{0}^{\prime \prime}=10^{-4}$. Scattering parameters for a two-dimensional microwave resonator coupled to a YIG thin-film stripe are also given using perturbation theory (c) compared to experimental data (d).

$\left|S_{11}\right|$ parameters obtained using perturbation theory at $\omega_{c}=\omega_{0}$ is given. Note that to obtain those it was necessary to consider dissipation for both cavity and magnetic systems - much like the case we have just discussed for a thin-film stripe. This can again be accounted for by making $\omega_{c}=\omega_{c}^{\prime}+j \omega_{c}^{\prime \prime}$ and $\omega_{0}=\omega_{0}^{\prime}+j \omega_{0}^{\prime \prime}$, where $\omega_{c}^{\prime \prime}$ and $\omega_{0}^{\prime \prime}$ are the dissipation in the cavity and in the magnetic sample, respectively.

The same principles outlined above can also be applied to more complex resonator systems, as is the case of two-dimensional resonators. In Fig. 4(c) we show the theoretical spectra for a thin-film YIG stripe coupled to a Niobium Nitride $(\mathrm{NbN})$ superconducting in-plane resonator. The YIG sample size is $5 \times 10 \times 50 \mu \mathrm{m}^{3}$ which can be used to obtain the eigenfrequencies as done in the previous section - using Eq. 14. For comparison, in Fig. 4(d) we show the experimental spectra for the same set up. Here, the YIG sample was integrated into the planar superconducting devices using a Xenon Plasma Focused Ion Beam (PFIB) and the spectra was measured at $3.2 \mathrm{~K}$ - the full sample preparation and measurement techniques are detailed in Ref. [49]. Since the magneti- sation of YIG is known to grow at low temperatures, in our calculations we have used $\mu_{0} M_{s}=0.270 \mathrm{~T}$ [50]. The experimental spectra is in excellent agreement with that modeled using perturbation theory shown in Fig. 4(c).

\section{APPLICABILITY TO THE ULTRASTRONG COUPLING REGIME}

So far we have discussed systems wherein strong coupling between microwaves and magnons takes place. Whilst this is a challenging task and there is still a lot to be investigated in these systems, the boundaries are already being pushed to achieve extremely high cooperativity at microwave frequencies. This is the case of the ultrastrong coupling regime which is when $g / \omega>0.1$ and it is also where approaches such as the rotating wave approximation begin to breakdown here [51]. Therefore, we will briefly investigate how our approach can be applied to one of these systems: a double-post, re-entrant microwave resonator coupled to a YIG sphere as investi- 


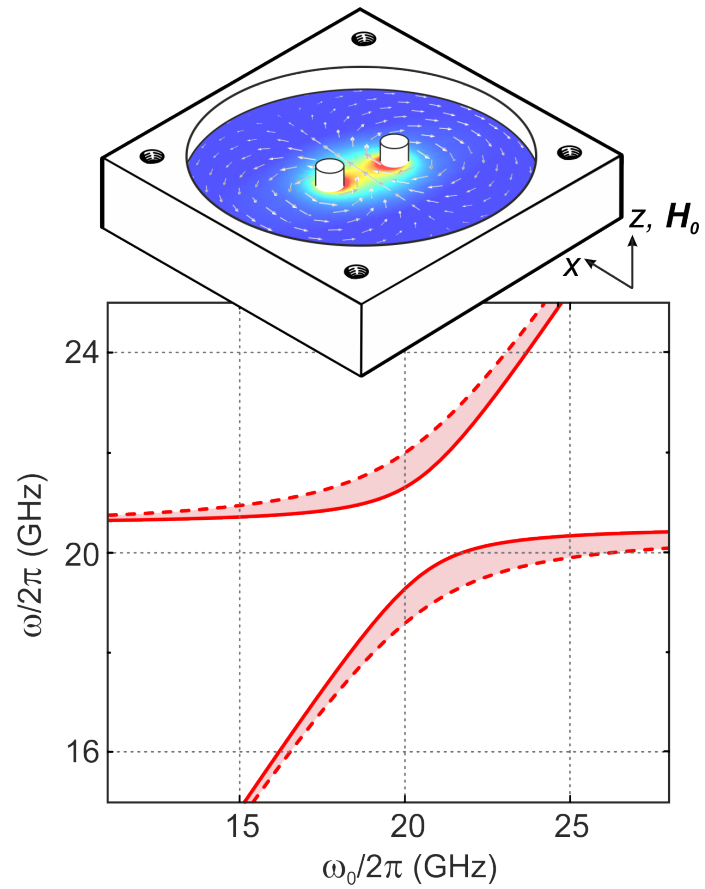

FIG. 5. Anti-crossing for the hybridisation between a YIG sphere and the the dark mode of a re-entrant resonator. The solid lines are the eigenfrequencies for a sample placed at the centre, between the posts. The dashed lines are the eigenfrequencies considering the strength of the field nearer the posts. The diagram above shows the resonator geometry and the field profile at $\omega_{c}=20.8 \mathrm{GHz}$ calculated using COMSOL 5.5 .

gated by Goryachev and co-workers [5]).

Re-entrant microwave resonators are threedimensional structures, built around a central post with an extremely small gap between one of the cavity walls and the post tip. This geometry allows for improved spatial separation between the cavitys oscillating electric and magnetic fields, $\mathbf{E}_{\mathbf{c}}$ and $\mathbf{h}_{\mathbf{c}}$ respectively. Double-post, re-entrant cavities, in particular, have been specifically designed to have a resonant mode wherein the $\mathbf{h}_{\mathbf{c}}$ field is focused into a relatively small region of the cavity. This is known as the cavity's "bright" mode.

Finite-element modeling was performed, using COMSOL 5.5, for a cavity of radius of $5.0 \mathrm{~mm}$ and height of $1.4 \mathrm{~mm}$ with the two posts in the centre having a radius of $0.4 \mathrm{~mm}$ each and are separated by $1.5 \mathrm{~mm}$. The distance between the posts and the lid is $73 \mu \mathrm{m}$ (see Ref. [5] for an extended discussion of experimental work using this cavity). The distribution of the magnetic field, $\mathbf{h}_{\mathbf{c}}$, for the cavity's "bright" mode is given in Fig. 5. From the electromagnetic solver, we also obtained the "bright" mode's resonance frequency $\omega_{c} / 2 \pi=20.8 \mathrm{GHz}$ as well as the following quantities: $W_{m}=W_{e}=6.43 \times 10^{-12} \mathrm{~J}$ and $W_{p}=2.0 \times 10^{-13} \mathrm{~J}$ at the centre of the cavity and $7.25 \times 10^{-13} \mathrm{~J}$ closer to the posts (both cases considering a YIG sphere of $0.8 \mathrm{~mm}$ diameter).

We can then plug these values into Eq. (12), along with $\mu_{0} M_{s}=0.255 \mathrm{~T}$ (value fitted by [5] for their experimental data obtained at $20 \mathrm{mK}$ ), to find the eigenfrequencies, as plotted in Fig. 5. The solid lines are for the sample placed at the centre of the cavity and the dashed lines were obtained using the values for $\mathbf{h}_{\mathbf{c}}$ closer to the posts. The shaded region shows how $\omega_{\text {gap }}$ can be tuned from the lower limit (solid lines) to the upper limit (dashed lines) by simply changing the intensity of $\mathbf{h}_{\mathbf{c}}$. These yield a gap, at $\omega_{c}=\omega_{0}$, of $\omega_{\text {gap }} / 2 \pi=2.1 \mathrm{GHz}$ (solid lines) and $4.0 \mathrm{GHz}$ (dashed lines). The value for the sample placed at the centre is, in fact, in excellent agreement with that reported by Goryachev and co-workers of approximately $2 \mathrm{GHz}[5]$. This agreement not only highlights how the theory can be successfully applied to ultrastrong coupling, but also how it can be used to tailor experiments. For instance, making the posts closer together in the reentrant cavity could allow for even stronger coupling, in which case perturbation theory could be employed to find the optimum ratio between the gap and sample size to obtain the strongest possible coupling.

\section{FINAL REMARKS}

To fully understand the electromagnetic behaviour of cavity-magnon hybrid systems we have employed a versatile and self-consistent theory which is an excellent tool to estimate the coupling strength $g$ (or width of $\omega_{\text {gap }}$ at $\omega_{c}=\omega_{0}$ ) without fitting parameters such as spin density from the strong to ultrastrong coupling regimes. This technique allows us to describe the direct interaction between the microwave excitation vector fields and magnetisation dynamics. The direction, profile, and intensity of microwave fields can dramatically alter magnoncavity hybridised states, i.e. cavity magnon-polaritons. The understanding presented here is particularly relevant for technological applications based on cavity spintronics. For instance, these systems are expected to aid bidirectional conversion between radio-frequency waves and light $[14,52]$. Moreover, as cavity magnonpolaritons can also couple with qubits [53], they are also expected to be used as an aid to quantum information processing $[8,9]$. In both cases, engineering as well as understanding the coupling are crucial steps to optimise the conversion and (or) information exchange.

In our work, through perturbation theory, we are able to predict as well as gain further insight into the nature of the coupling between microwave cavities and magnons in a rigorous manner for any resonator with any field configuration and for any geometry of the sample (e.g. spheres or thin films, pillars, etc). Our theory is particularly relevant, not only from a fundamental point of view, but also practically, as in order to engineer and optimise cavity spintronic devices the behaviour of the coupling must be fully understood. While the constant $g$ is more often obtained from experimental data and incorporated into models, such as the circuit model [24], 
there have been efforts to describe the coupling with no fitting parameters $[6,38]$. This however, is done through phenomenological oscillator models where the total spin number is key. The work presented here employs the magnetic susceptibility of a sphere and rectangular prism to find the magnon-cavity coupling. The magnetic susceptibility can be found for vastly more complicated magnetic systems, where exchange interactions and dipolar interactions are important to consider. This involves solving the Landau-Lifshitz equation numerically for an array of spins, rather than a macrospin as was done here. Accurate prediction is therefore possible for the coupling strength between electromagnetic cavity waves and magnons in samples with complicated shapes or spin orderings. Our findings show excellent agreement with recently published works towards miniaturisation of hybrid systems at milli-Kelvin temperatures and provide an avenue to predict the coupling to not only extremely low damping magnetic films such as YIG [49], but also to highly damped metallic thin films $[43,44]$.

\section{ACKNOWLEDGMENTS}

We would like to thank Robert E. Camley, Tim Wolz and Isabella Boventer for their most useful discussions as well as their comments on the manuscript. This work was supported by the European Research Council (ERC) under the Grant Agreement 648011, the Initiative and Networking Fund of the Helmholtz Association, the Leverhulme Trust and the University of Glasgow through LKAS funds. D. A. Bozhko acknowledges support from the Alexander von Humboldt Foundation. R. Holland was supported by the Engineering and Physical Sciences Research Council (EPSRC) through the Vacation Internships Scheme.

\section{ASSOCIATED CONTENT}

A python code reproducing the theory employed above is provided in the following repository:

https://github.com/UofG-

PhtonicsSpinElectronics/Perturbation-Theory-for-

Cavity-Magnon-Polaritons

\section{Appendix A: Full Susceptibility Tensor}

Further to $\chi_{x x}-$ which was given in the main text as Eq. (4) - the other components of the susceptibility tensor, $\overleftrightarrow{\chi}_{m}(\omega)$, given in Eq. 3 are:

$$
\begin{array}{r}
\chi_{y y}(\omega)=\frac{\chi_{b}}{1-\left(\omega / \omega_{0}\right)^{2}}, \\
\chi_{y x}(\omega)=-\chi_{x y}(\omega)=\frac{j \omega \omega_{m}}{\omega_{0}^{2}\left[1-\left(\omega / \omega_{0}\right)^{2}\right]},
\end{array}
$$

where

$$
\chi_{b}=\frac{M_{s}}{H_{0}+\left(D_{y}-D_{z}\right) M_{s}} .
$$

\section{Appendix B: Fields in a Rectangular Cavity Resonator}

The rectangular cavity resonator shown in Fig. 2(b) includes height $(a=54 \mathrm{~mm})$, length $(b=36 \mathrm{~mm})$, and width $(c=7.5 \mathrm{~mm})$. We have concentrated on a $\mathrm{TE}_{m n}$ propagation mode where the subscripts $m$ and $n$ come from the wave propagation modes and represent the changing cycles along $x$ and $y$.

On the basis of our discussion on a TE propagation mode of a rectangular cavity and the boundary conditions, the field distribution of these modes can be written as:

$$
\begin{array}{r}
h_{c x}=j A \frac{\kappa_{0 y}}{\omega_{c} \mu_{0}} \sin \kappa_{0 x} x \cos \kappa_{0 y} y \\
h_{c y}=-j A \frac{\kappa_{0 x}}{\omega_{c} \mu_{0}} \cos \kappa_{0 x} x \sin \kappa_{0 y} y
\end{array}
$$

with

$$
\kappa_{0 x}=\frac{m \pi}{a}, \kappa_{0 y}=\frac{n \pi}{b}, \kappa_{0 x}^{2}+\kappa_{0 y}^{2}=\kappa_{0}^{2}=\omega^{2} \varepsilon_{0} \mu_{0},
$$

and $A$ is an amplitude constant related to the microwave power into the cavity with the appropriate units. Since this affects the amplitude of the fields, but does not affect its distribution, here we simply taken it as unity.

Note that these field profiles can also be calculated using electromagnetic solver such as COMSOL or HFSS.

\section{Appendix C: General form of $W_{p}$}

In the most general case, where the excitation vector fields lie in both $x$ and $y$ directions, the numerator of Eq. (9) is now given by:

$$
\begin{aligned}
\int_{\delta v} \mu_{0}\left[\chi_{x x}(\omega)\left|h_{c x}\right|^{2}+\chi_{y y}(\omega)\left|h_{c y}\right|^{2}\right. \\
+j \chi_{x y}(\omega)\left(\left(h_{c y} h_{c x}^{*}-h_{c x} h_{c y}^{*}\right)\right] d v
\end{aligned}
$$

or simply: 


$$
\begin{aligned}
\int_{\delta v} \mu_{0} \frac{\omega_{0} \omega_{m}}{\omega_{0}^{2}-\omega^{2}}\left[\left|h_{c x}\right|^{2}+\left|h_{c y}\right|^{2}\right. & \\
& +j \frac{\omega}{\omega_{0}}\left(\left(h_{c y} h_{c x}^{*}-h_{c x} h_{c y}^{*}\right)\right] d v
\end{aligned}
$$

Thus, in the limit close to $\omega=\omega_{0}$, we can write $W_{p}$ as simply:

$$
W_{p}=\int_{\delta v}\left[\left|h_{c x}\right|^{2}+\left|h_{c y}\right|^{2}+j\left(\left(h_{c y} h_{c x}^{*}-h_{c x} h_{c y}^{*}\right)\right] d v\right.
$$

making the current form of Eqs.(11)-(13) does not change, apart from the value of $W_{p}$ to incorporate the different direction of $\mathbf{h}_{\mathbf{c}}$. Note that these equations hold true for a sphere only. They would need to be modified with the relevant susceptibility tensor components for other shaped systems considering the effects of demagnetising fields. For instance, for a rectangular prism, as that shown in Fig. 1(b), one can simply take Eqs. (4),(A1a), and (A1b) and plug them into Eq. (C1) to find the appropriate numerator of Eq. (9).

\section{Appendix D: Experimental Setup Details}

For the three-dimensional rectangular resonator measurements, microwave signals were supplied by port 1 of a Rohde \& Schwarz ZVA 40 vector network analyser (VNA) and signals reflected from or transmitted through the cavity were sent to port 2 of the VNA. The capacitive coupling to the cavity was tuned by adjusting the length of the SMA connector contacts, which extended into the body of the cavity.
[1] E. M. Purcell, H. C. Torrey, and R. V. Pound, Resonance absorption by nuclear magnetic moments in a solid, Phys. Rev. 69, 37 (1946).

[2] H. Walther, B. T. Varcoe, B.-G. Englert, and T. Becker, Cavity quantum electrodynamics, Reports on Progress in Physics 69, 1325 (2006).

[3] J. H. E. Griffiths, Anomalous high-frequency resistance of ferromagnetic metals, Nature 158, 670 (1946).

[4] C. Kittel, On the theory of ferromagnetic resonance absorption, Physical Review 73, 155 (1948).

[5] M. Goryachev, W. G. Farr, D. L. Creedon, Y. Fan, M. Kostylev, and M. E. Tobar, High-cooperativity cavity qed with magnons at microwave frequencies, Phys. Rev. Applied 2, 054002 (2014).

[6] X. Zhang, C.-L. Zou, L. Jiang, and H. X. Tang, Strongly coupled magnons and cavity microwave photons, Phys. Rev. Lett. 113, 156401 (2014).

[7] D. Zhang, X.-M. Wang, T.-F. Li, X.-Q. Luo, W. Wu, F. Nori, and J. You, Cavity quantum electrodynamics with ferromagnetic magnons in a small yttrium-irongarnet sphere, npj Quantum Information 1, 1 (2015).

[8] Y. Tabuchi, S. Ishino, A. Noguchi, T. Ishikawa, R. Yamazaki, K. Usami, and Y. Nakamura, Coherent coupling between a ferromagnetic magnon and a superconducting qubit, Science 349, 405 (2015).

[9] D. Lachance-Quirion, Y. Tabuchi, A. Gloppe, K. Usami, and Y. Nakamura, Hybrid quantum systems based on magnonics, Applied Physics Express 12, 070101 (2019).

[10] X. Zhang, C.-L. Zou, N. Zhu, F. Marquardt, L. Jiang, and H. X. Tang, Magnon dark modes and gradient memory, Nature communications 6, 8914 (2015).

[11] N. Crescini, D. Alesini, C. Braggio, G. Carugno, D. Di Gioacchino, C. S. Gallo, U. Gambardella, C. Gatti, G. Iannone, G. Lamanna, C. Ligi, A. Lombardi, A. Ortolan, S. Pagano, R. Pengo, G. Ruoso, C. C. Speake, and L. Taffarello, Operation of a ferromagnetic axion haloscope at $m_{a}=58 \mu \mathrm{ev}$, The European Physical Journal C 78, 703 (2018).
[12] G. Flower, J. Bourhill, M. Goryachev, and M. E. Tobar, Broadening frequency range of a ferromagnetic axion haloscope with strongly coupled cavitymagnon polaritons, Physics of the Dark Universe 25, 100306 (2019).

[13] N. Crescini, C. Braggio, G. Carugno, A. Ortolan, and G. Ruoso, Cavity magnon polariton based precision magnetometry, Applied Physics Letters 117, 144001 (2020), https://doi.org/10.1063/5.0024369.

[14] R. Hisatomi, A. Osada, Y. Tabuchi, T. Ishikawa, A. Noguchi, R. Yamazaki, K. Usami, and Y. Nakamura, Bidirectional conversion between microwave and light via ferromagnetic magnons, Phys. Rev. B 93, 174427 (2016).

[15] D. Zhang, X.-Q. Luo, Y.-P. Wang, T.-F. Li, and J. You, Observation of the exceptional point in cavity magnonpolaritons, Nature communications 8, 1368 (2017).

[16] M. Harder, Y. Yang, B. M. Yao, C. H. Yu, J. W. Rao, Y. S. Gui, R. L. Stamps, and C.-M. Hu, Level attraction due to dissipative magnon-photon coupling, Phys. Rev. Lett. 121, 137203 (2018).

[17] S. Kaur, B. M. Yao, J. W. Rao, Y. S. Gui, and C.-M. $\mathrm{Hu}$, Voltage control of cavity magnon polariton, Applied Physics Letters 109, 032404 (2016), https://doi.org/10.1063/1.4959140.

[18] H. Maier-Flaig, M. Harder, S. Klingler, Z. Qiu, E. Saitoh, M. Weiler, S. Geprgs, R. Gross, S. T. B. Goennenwein, and H. Huebl, Tunable magnon-photon coupling in a compensating ferrimagnetfrom weak to strong coupling, Applied Physics Letters 110, 132401 (2017), https://doi.org/10.1063/1.4979409.

[19] B. Bhoi, B. Kim, S.-H. Jang, J. Kim, J. Yang, Y.-J. Cho, and S.-K. Kim, Abnormal anticrossing effect in photonmagnon coupling, Phys. Rev. B 99, 134426 (2019).

[20] X. Zhang, A. Galda, X. Han, D. Jin, and V. M. Vinokur, Strong coupling-enabled broadband nonreciprocity (2019), arXiv:1910.14117 [cond-mat.meshall].

[21] I. Boventer, M. Klui, R. Macêdo, and M. Weides, Steering between level repulsion and attraction: broad tunability 
of two-port driven cavity magnon-polaritons, New Journal of Physics 21, 125001 (2019).

[22] I. Boventer, C. Dörflinger, T. Wolz, R. Macêdo, R. Lebrun, M. Kläui, and M. Weides, Control of the coupling strength and linewidth of a cavity magnon-polariton, Phys. Rev. Research 2, 013154 (2020).

[23] T. Wolz, A. Stehli, A. Schneider, I. Boventer, R. Macêdo, A. V. Ustinov, M. Kläui, and M. Weides, Introducing coherent time control to cavity magnon-polariton modes, Communications Physics 3, 1 (2020).

[24] M. Harder, L. Bai, C. Match, J. Sirker, and C. Hu, Study of the cavity-magnon-polariton transmission line shape, Science China Physics, Mechanics \& Astronomy 59, 117511 (2016).

[25] I. Proskurin, R. Macêdo, and R. L. Stamps, Microscopic origin of level attraction for a coupled magnon-photon system in a microwave cavity, New Journal of Physics 21, 095003 (2019).

[26] Y. Cao and P. Yan, Exceptional magnetic sensitivity of $\mathcal{P} \mathcal{T}$-symmetric cavity magnon polaritons, Phys. Rev. B 99, 214415 (2019).

[27] V. Cherepanov, I. Kolokolov, and V. L'vov, The saga of yig: Spectra, thermodynamics, interaction and relaxation of magnons in a complex magnet, Physics Reports 229, 81 (1993).

[28] A. A. Serga, A. V. Chumak, and B. Hillebrands, YIG magnonics, Journal of Physics D: Applied Physics 43, 264002 (2010).

[29] A. G. Gurevich and G. A. Melkov, Magnetization oscillations and waves (CRC press, Inc, Boca Raton, Florida, 1996) Chap. 1.

[30] W. Brown, Magnetostatic Principles in ferromagnetism, (Series of monographs on selected topics in solid state physics) (North-Holland Publishing Company, 1962).

[31] A. Aharoni, Demagnetizing factors for rectangular ferromagnetic prisms, Journal of Applied Physics 83, 3432 (1998), https://doi.org/10.1063/1.367113.

[32] M. Gppl, A. Fragner, M. Baur, R. Bianchetti, S. Filipp, J. M. Fink, P. J. Leek, G. Puebla, L. Steffen, and A. Wallraff, Coplanar waveguide resonators for circuit quantum electrodynamics, Journal of Applied Physics 104, 113904 (2008), https://doi.org/10.1063/1.3010859.

[33] R. Camley, Nonreciprocal surface waves, Surface Science Reports 7, 103 (1987).

[34] R. Macêdo and R. E. Camley, Engineering terahertz surface magnon-polaritons in hyperbolic antiferromagnets, Phys. Rev. B 99, 014437 (2019).

[35] R. Macdo, Chapter two - tunable hyperbolic media: Magnon-polaritons in canted antiferromagnets (Academic Press, 2017) pp. $91-155$.

[36] H. How, Magnetic microwave devices. in encyclopedia of rf and microwave engineering, k. chang (ed.), in (John $\frac{\text { Encyclopedia of RF and Microwave Engineering }}{\text { Wiley \& Sons, Inc., Hobo- }}$ ken, New Jersey., 2005) pp. 2425-2461, https://onlinelibrary.wiley.com/doi/pdf/10.1002/047165450

[37] A. J. Baden-Fuller, Ferrites at microwave frequencies, 23 (Peter Perogrinus Ltd., London, United Kingdom, 1987).

[38] J. Bourhill, V. Castel, A. Manchec, and G. Cochet, Universal characterization of cavity-magnon polariton coupling strength verified in modifiable microwave cavity, Journal of Applied Physics 128, 073904 (2020).

[39] R. Waldron, Theory of the measurement of the elements of the permeability tensor of a ferrite by means of a res- onant cavity, Proceedings of the IEE-Part B: Radio and Electronic Engineering 104, 307 (1957).

[40] D. Pozar, Microwave Engineering, 4th Edition (John Wiley \& Sons, Inc, New York, 2011) Chap. 6.

[41] R. Miller, T. E. Northup, K. M. Birnbaum, A. Boca, A. D. Boozer, and H. J. Kimble, Trapped atoms in cavity QED: coupling quantized light and matter, Journal of Physics B: Atomic, Molecular and Optical Physics 38, S551 (2005).

[42] See Supplemental Material at [URL will be inserted by publisher] for details on the evaluation of perturbation theory for increasing the volume of the sample within the resonator. We compare the analytical results with COMSOL 5.5 simulations obtaining excellent agreement.

[43] J. T. Hou and L. Liu, Strong coupling between microwave photons and nanomagnet magnons, Phys. Rev. Lett. 123, 107702 (2019).

[44] Y. Li, T. Polakovic, Y.-L. Wang, J. Xu, S. Lendinez, Z. Zhang, J. Ding, T. Khaire, H. Saglam, R. Divan, J. Pearson, W.-K. Kwok, Z. Xiao, V. Novosad, A. Hoffmann, and W. Zhang, Strong coupling between magnons and microwave photons in on-chip ferromagnetsuperconductor thin-film devices, Phys. Rev. Lett. 123, 107701 (2019).

[45] See Supplemental Material at [URL will be inserted by publisher] for details on the calculation of the field profile and stored energy for a CPW resonator using COMSOL 5.5 .

[46] D. Han, Y. Kim, and M. Kwon, Two port cavity q measurement using scattering parameters, Review of Scientific Instruments 67, 2179 (1996), https://doi.org/10.1063/1.1147034.

[47] A. Luiten, Q-factor measurements. in encyclopedia of $\mathrm{rf}$ and microwave engineering, k. chang (ed.), in Encyclopedia of RF and Microwave Engineering (John Wiley \& Sons, Inc., Hoboken, New Jersey., 2005) pp. 3948-3964, https://onlinelibrary.wiley.com/doi/pdf/10.1002/0471654507.eme332

[48] S. Probst, F. B. Song, P. A. Bushev, A. V. Ustinov, and M. Weides, Efficient and robust analysis of complex scattering data under noise in microwave resonators, Review of Scientific Instruments 86, 024706 (2015), https://doi.org/10.1063/1.4907935.

[49] P. Baity and et. al., Strong magnon-photon coupling onchip with yig in the zero-temperature limit, Unpublished (2020).

[50] I. Boventer, M. Pfirrmann, J. Krause, Y. Schön, M. Kläui, and M. Weides, Complex temperature dependence of coupling and dissipation of cavity magnon polaritons from millikelvin to room temperature, Phys. Rev. B 97, 184420 (2018).

[51] G. Flower, M. Goryachev, J. Bourhill, and M. E. Tobar, Experimental implementations of cavity-magnon systems: from ultra strong coupling to applications in precision measurement, New Journal of Physics 21, 095004 7.eme(2019).

[52] N. J. Lambert, A. Rueda, F. Sedlmeir, and H. G. L. Schwefel, Coherent conversion between microwave and optical photonsan overview of physical implementations, Advanced Quantum Technologies 3, 1900077 (2020), https://onlinelibrary.wiley.com/doi/pdf/10.1002/qute.201900077.

[53] D. Lachance-Quirion, S. P. Wolski, Y. Tabuchi, S. Kono, K. Usami, and Y. Nakamura, Entanglementbased single-shot detection of a single magnon with 
a superconducting qubit, Science 367, 425 (2020), https://science.sciencemag.org/content/367/6476/425.full.pdf. 